A. Batou - C. Soize

\title{
Identification of stochastic loads applied to a non-linear dynamical system using an uncertain computational model and experimental responses
}

Received: date / Accepted: date

\begin{abstract}
The paper is devoted to the identification of stochastic loads applied to a non-linear dynamical system for which experimental dynamical responses are available. The identification of the stochastic load is performed using a simplified computational non-linear dynamical model containing both model uncertainties and data uncertainties. Uncertainties are taken into account in the context of the probability theory. The stochastic load which has to be identified is modelled by a stationary non Gaussian stochastic process for which the matrix-valued spectral density function is uncertain and is then modelled by a matrix-valued random function. The parameters to be identified are the mean value of the random matrix-valued spectral density function and its dispersion parameter. The identification problem is formulated as two optimisation problems using the computational stochastic model and experimental responses. A validation of the theory proposed is presented in the context of tubes bundles in Pressurized Water Reactors.
\end{abstract}

Keywords non-linear dynamics $\cdot$ random loads $\cdot$ inverse problem

\section{Introduction}

The present research has been developed in the context of the dynamical analysis of the tubes bundles in Pressurized Water Reactors. The tubes are excited by a turbulent flow which induces a non-linear dynamical response of the dynamical system made up of a structure coupled with the fluid. The objective is to identify a mathematical model of the stochastic loads applied to the tubes bundles and induced by the turbulent flow using both experimental responses of the real non-linear dynamical system and a simplified computational non-linear dynamical model. In general, for such a complex mechanical system, only a few experiments are available and in many cases, the measurements are available only for one configuration of the dynamical system. Consequently, one will assume that measurements are available for one configuration. In addition, the real system under consideration is

\footnotetext{
A. Batou

(1) Université Paris-Est, Laboratoire Modélisation et Simulation Multi échelle, FRE3160 CNRS,

5 bd Descartes, 77454 Marne-la-Vallee, France

(2) EDF, Laboratoire Mécanique des Structures Industrielles Durables, UMR2832 CNRS,

1 avenue du Géneral de Gaulle, 92140 Clamart, France

C. Soize

Université Paris-Est, Laboratoire Modélisation et Simulation Multi échelle, FRE3160 CNRS,

5 bd Descartes, 77454 Marne-la-Vallee, France Tel.: +33160957661

Fax: +33160957799

E-mail: christian.soize@univ-paris-est.fr
} 
made up of several hundreds of tubes and several grids. The dynamical behaviour of any tube in a free-free configuration is linear. The non-linearities are induced by the shocks between the tubes and the grids. In the frequency band of analysis for which the stochastic loads have to be identified, there are between ten thousand and twenty thousand elastic modes for the linearized dynamical system. In this condition a simplified computational non-linear dynamical model has to be introduced for that the identification of the stochastic loads can effectively be done. For the identification of the stochastic loads, the use of a simplified computational model induces model uncertainties. A nonparametric probabilistic approach is then used to take into account both data uncertainties and model uncertainties

Such a problem has already been studied in modelling the real non-linear dynamical system by a simplified linear dynamical system $([7],[8],[9])$. In this paper we take into account nonlinearities and also uncertainties both in the computational model and in the stochastic process representing the stochastic loads.

In the context above, a methodology is presented for identifying such stochastic loads. The real dynamical system (real tubes bundles) is replaced by a reference non-linear dynamical system made up of five tubes and three grids. This reference system is representative of the real system and a computational model, defined as the reference computational model, is developed. This reference computational model allows the responses of the reference system to be simulated. Therefore, the experimental responses of the tubes related to one configuration of the real system are replaced by the numerical responses of the reference system calculated with the reference computational model. The simplified computational model is derived from the reference computational model .

In a first step, the probability model of uncertainties is identified in the simplified computational model using the reference computational model and the maximum likelihood method. We then deduce a stochastic simplified computational model which allows a robust identification of stochastic loads to be carried out with respect to uncertainties in the dynamical system.

The second step is devoted to the stochastic inverse problem consisting in identifying the stochastic loads. The stochastic loads used in the simplified computational model are represented by a vectorvalued centred stationary Gaussian stochastic process. Such a stochastic process is then completely defined by a matrix-valued spectral density function. The use of a rough spatial discretization of the random field in the simplified computational model introduces uncertainties in the stochastic process which models the stochastic loads. These uncertainties are then taken into account in introducing a probabilistic model for the matrix-valued spectral density function which becomes a random quantity which has to be constructed and identified. The identification of the stochastic loads then corresponds (1) to the identification of the mean value of the random matrix-valued spectral density function in the frequency band of analysis and (2) to the identification of the dispersion parameter introduced in the probability model of uncertainties and allowing the level of uncertainties to be controlled. We then have to perform the identification of an uncertain model of the stochastic loads using an uncertain simplified computational model of a non-linear dynamical system and using responses of the reference computational model. This problem is formulated as a stochastic optimization problem. It should be noted that this inverse stochastic problem is formulated and solved for a non-linear stochastic dynamical system including a probability model of uncertainties for the stochastic loads and for the system.

Sections 2 and 3 respectivly deals with the construction of the reference computational model and the simplified computational model. In the section 3, the probabilistic model of the stochastic loads is introduced. The section 4 is devoted to the identification of the stochastic load introduced in section 3 using numerical response simulations of the reference model and the stochastic model of the simplified computational model. The last section presents the application of this reseach using a representative real system.

\section{Reference computational model}

In this section, we introduce a reference computational model for which the responses will be considered as the experimental responses and will be used to identify the stochastic simplified computational model that will be introduced in the next session. 


\subsection{Transient dynamical response of the reference computational model}

Let $\Omega$ be the domain of a three dimensional damped structure having a non-linear behaviour (the nonlinearities are not distributed but are localized). The structure is fixed on the part $\Gamma_{0}$ of the boundary $\Gamma$ of $\Omega$. Let $\mathbf{u}^{r e f}(t)$ be the vector of the $n$ degrees of freedom at time $t$. Let $\left[\mathbb{M}^{r e f}\right]$, $\left[\mathbb{D}^{\text {ref }}\right]$ and $\left[\mathbb{K}^{r e f}\right]$ be respectively the mass, damping and stiffness matrices of the linear part of the finite element model. Since there are no rigid body displacements, these three matrices are positive definite. Let $\tilde{\mathbf{f}}^{r e f}(t)$ be the vector of the external loads applied to the structure and let $\tilde{\mathbf{f}}^{N L}\left(\mathbf{u}^{\text {ref }}(t), \dot{\mathbf{u}}^{\text {ref }}(t)\right)$ be the vector of the non-linear forces induced by the localized non-linearities. Let $[\Phi]$ be $(n \times m)$ matrix whose columns are the $m$ structural modes $\varphi_{1}, \ldots, \varphi_{m}$ of the linear structure without the non-linearities corresponding to the $m$ eigenfrequencies $0<\omega_{1} \leq \ldots \leq \omega_{m}$. The non-linear dynamical equation is projected on the the basis represented by $[\Phi]$. Therefore, the displacement vector at time $t$ is written as $\mathbf{u}^{\text {ref }}(t)=[\Phi] \mathbf{q}^{\text {ref }}(t)$ in which $\mathbf{q}^{r e f}(t)$ is the vector of the $m$ generalized coordinates. Such a projection introduces the positive definite matrices $\left[M^{\text {ref }}\right]=[\Phi]^{T}\left[\mathbb{M}^{\text {ref }}\right][\Phi],\left[D^{\text {ref }}\right]=[\Phi]^{T}\left[\mathbb{D}^{\text {ref }}\right][\Phi]$ and $\left[K^{r e f}\right]=[\Phi]^{T}\left[\mathbb{K}^{\text {ref }}\right][\Phi]$. The function $t \mapsto \mathbf{q}^{r e f}(t)$ is the solution of the following non-linear dynamical equation,

$$
\left[M^{r e f}\right] \ddot{\mathbf{q}}^{r e f}(t)+\left[D^{r e f}\right] \dot{\mathbf{q}}^{r e f}(t)+\left[K^{r e f}\right] \mathbf{q}^{r e f}(t)+\mathcal{F}^{N L}\left(\mathbf{u}^{r e f}(t), \dot{\mathbf{u}}^{r e f}(t)\right)=\mathcal{F}^{r e f}(t), t \in[0, T],
$$

with the initial conditions

$$
\mathbf{q}^{r e f}(0)=\dot{\mathbf{q}}^{r e f}(0)=0,
$$

where $\mathcal{F}^{N L}\left(\mathbf{u}^{r e f}(t), \dot{\mathbf{u}}^{r e f}(t)\right)=[\Phi]^{T} \tilde{\mathbf{f}}^{N L}\left(\mathbf{u}^{r e f}(t), \dot{\mathbf{u}}^{\text {ref }}(t)\right)$ and $\mathcal{F}^{r e f}(t)=[\Phi]^{T} \tilde{\mathbf{f}}^{r e f}(t)$.

\subsection{Decomposition in one linear subsystem and one non-linear subsystem}

The domain $\Omega$ is decomposed in two subdomains, the subdomain $\Omega_{r e f}^{A}$ which corresponds to a nonlinear subsystem made up of one part of the structure containing the localized non-linearities and the subdomain $\Omega_{r e f}^{B}$ which corresponds to a linear subsystem made up of the second part of the structure and which has a linear behaviour. Each uncoupled subsystem is considered as fixed and therefore does not have rigid body displacement. These two subsytems are coupled on the coupling interface $\Gamma_{C}$. The finite element model of the linear subsytem $\Omega_{\text {ref }}^{B}$ is analyzed in the frequency band of analysis $\mathcal{B}=\left[-\omega_{\max }, \omega_{\max }\right]$. Let $\left[\mathbb{A}^{B, r e f}(\omega)\right]$ be the dynamic stiffness matrix of this linear subsystem with free coupling interface such that

$$
\left[\mathbb{A}^{B, r e f}(\omega)\right]=-\omega^{2}\left[\mathbb{M}^{B, r e f}\right]+i \omega\left[\mathbb{D}^{B, r e f}\right]+\left[\mathbb{K}^{B, r e f}\right]
$$

where $\left[\mathbb{M}^{B, \text { ref }}\right],\left[\mathbb{D}^{B, r e f}\right]$ and $\left[\mathbb{K}^{B, r e f}\right]$ are the mass, damping and stiffness matrices which are positive definite. Introducing the vector $\mathbf{u}_{p}^{B, r e f}(\omega)$ of the $n_{p}$ internal DOF and the vector $\mathbf{u}_{c}^{B, r e f}(\omega)$ of the $n_{c}$ coupling DOF on the interface, the equation of the reference computational model for the subsystem $\Omega_{B_{\text {ref }}}$ is written as

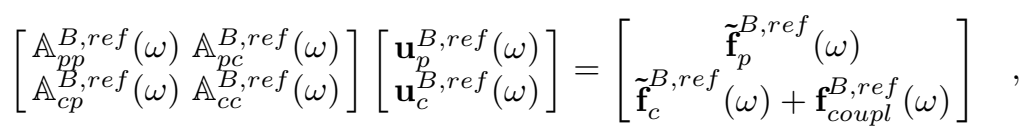

in which $\tilde{\mathbf{f}}_{p}^{B, r e f}(\omega)$ and $\tilde{\mathbf{f}}_{c}^{B, r e f}(\omega)$ are due to the external loads applied to the subsystem and where $\mathbf{f}_{\text {coupl }}^{B, r e f}(\omega)$ is the internal force applied to the coupling interface $\Gamma_{C}$.

A reduction of the linear subsystem is performed using the Craig Bampton method [5],introducing the change of coordinates,

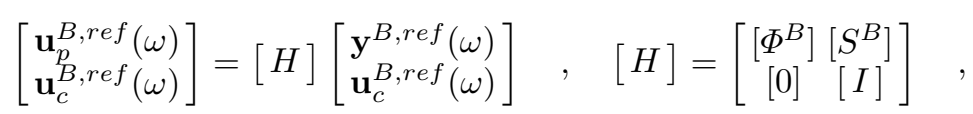

where $\left[\Phi^{B}\right]$ is a $\left(n_{p} \times N_{r}\right)$ matrix whose columns are $N_{r}$ structural modes of the reference computational model with a fixed coupling interface, $\left[S^{B}\right]=\left[\mathbb{K}_{p p}^{B, r e f}\right]^{-1}\left[\mathbb{K}_{p c}^{B, r e f}\right]$ is a $\left(n_{p} \times n_{c}\right)$ matrix related to the 
static boudary functions and $[I]$ is a $\left(n_{c} \times n_{c}\right)$ unnity matrix. $\mathbf{y}^{B, r e f}(\omega)$ is the vector of the $N_{r}$ generalized coordinates. Then, the reduced stiffness matrix is written as

$$
\left[A^{B, r e f}(\omega)\right]=-\omega^{2}\left[M^{B, r e f}\right]+i \omega\left[D^{B, r e f}\right]+\left[K^{B, r e f}\right],
$$

where $\left[M^{B, \text { ref }}\right]=[H]^{T}\left[\mathbb{M}^{B, \text { ref }}\right][H],\left[D^{B, \text { ref }}\right]=[H]^{T}\left[\mathbb{D}^{B, \text { ref }}\right][H]$ and $\left[K^{B, \text { ref }}\right]=[H]^{T}\left[\mathbb{K}^{B, \text { ref }}\right][H]$ are positive definite matrices. The block decomposition of the reduced dynamical stiffness matrix related to the generalized coordinates $\mathbf{y}^{B}(\omega)$ and the coupling DOF $\mathbf{u}_{c}^{B}(\omega)$ is written as

$$
\left[A^{B, r e f}(\omega)\right]=\left[\begin{array}{l}
A_{y y}^{B, r e f}(\omega) A_{y c}^{B, r e f}(\omega) \\
A_{c y}^{B, r e f}(\omega) A_{c c}^{B, r e f}(\omega)
\end{array}\right] .
$$

In order to perform the identification of stochastic simplified computational model, for the reference computational model, below we introduce an observation related to $\left[Z^{B, r e f}(\omega)\right]=\left[\left[A_{c c}^{B, r e f}(\omega)\right]-\right.$ $\left.\left[A_{c y}^{B}(\omega)\right]\left[A_{y y}^{B, r e f}(\omega)\right]^{-1}\left[A_{y c}^{B, r e f}(\omega)\right]\right]$ which corresponds to the condensed dynamical stiffness matrix of the linear subsystem on the coupling interface of the reference computational model. For all $\omega$ fixed in the frequency band $\mathcal{B}$, the matrix $\left[Z^{B, r e f}(\omega)\right]$ is invertible. The observation is then the finite positive real number $J^{r e f}$ defined by

$$
J^{r e f}=\int_{\mathcal{B}}\left\|\left[Z^{B, r e f}(\omega)\right]^{-1}\right\|_{F}^{2} d \omega
$$

\section{Stochastic non-linear simplified computational model, including system uncertainties, and identification}

In this part, the mean model of the non-linear simplified computational model system is introduced. Then, the probabilistic nonparametric approach will be used to take into account data uncertainties and model uncertainties in the linear subsystem of the simplified computational model. Then, the dispersion parameters controlling the dispersion on the mass, damping and stiffness matrices will be identified using the maximum likelihood method. Finally we proceed to the coupling of the two subsystems of the simplified model (the stochastic linear subsystem and the deterministic nonlinear subsystem) in order to compute its stochastic response. It should be noted that the probabilistic model of uncertainties is only introduced in the linear subsystem. It is assumed that the mean model of the non-linear subsystem is representative and that both data uncertainties and model uncertainties are negligible. Therefore, if such a hypothesis was not verified, a non-parametric probabilistic model of uncertainties could always be implemented without any difficulty in this non-linear subsystem [12].

\subsection{Stochastic linear subsystem of the simplified computational model}

The simplified computational model is constructed from the reference computational model. The difference between the two models is in the linear subsytem. Indeed, the linear subsytem of the simplified model is derived from the linear part of the reference model. The non-linear subsystems of the two models are the same (see Fig. 1). And consequently, the degrees of freedom on the coupling interface are the same.
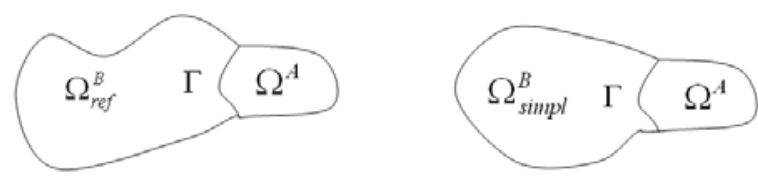

Fig. 1 Reference model (left) and simplified model (right). 


\subsubsection{Mean reduced linear subsystem of the simplified computational model}

Using the same reduction method as the one used for the linear subsystem of the reference model, we obtain the following mean reduced dynamical stiffness matrix for the linear subsystem of the simplified model

$$
\left[\underline{A}^{B}(\omega)\right]=-\omega^{2}\left[\underline{M}^{B}\right]+i \omega\left[\underline{D}^{B}\right]+\left[\underline{K}^{B}\right],
$$

where $\left[\underline{M}^{B}\right],\left[\underline{D}^{B}\right]$ and $\left[\underline{K}^{B}\right]$ are respectively the mean reduced positive definite mass, damping and stiffness matrices of the linear subsystem of the mean reduced computational simplified model. $\underline{\mathbf{y}}^{B}(\omega)$ is the vector of the $N$ mean generalized coordinates and $\underline{\mathbf{u}}_{c}^{B}(\omega)$ is the vector of the $n_{c}$ mean coupling DOFs. Then, the block decomposition of the reduced stiffness matrix related to the generealized coordinates $\underline{\mathbf{y}}^{B}(\omega)$ and the coupling DOFs $\underline{\mathbf{u}}_{c}^{B}(\omega)$ is written as

$$
\left[\underline{A}^{B}(\omega)\right]=\left[\begin{array}{ll}
\underline{A}_{y y}^{B}(\omega) & \underline{A}_{y c}^{B}(\omega) \\
\underline{A}_{c y}^{B}(\omega) & \underline{A}_{c c}^{B}(\omega)
\end{array}\right] .
$$

\subsubsection{System uncertainties modeling using the non-parametric probabilistic approach}

The non-parametric probabilistic approach is used to take into account both model uncertainties and data uncertainties in the dynamical system. This approach has recently been introduced (see [1], [2]) and consists in replacing the matrices of reduced mean model by random matrices for which the probability distributions are constructed by using the maximum entropy principle with constraints defined by the available information. Such an approach has been validated for different cases. In particular, non-parametric probabilistic approach has simultaneously been used ([3])with substructuring technics. Let $n=N+n_{c}$. Therefore, the mean reduced dynamical stiffness matrix $\left[\underline{A}^{B}(\omega)\right]$ is replaced by the $n \times n$ complex random matrix $\left[\mathbf{A}^{B}(\omega)\right]$ written as

$$
\left[\mathbf{A}^{B}(\omega)\right]=-\omega^{2}\left[\mathbf{M}^{B}\right]+i \omega\left[\mathbf{D}^{B}\right]+\left[\mathbf{K}^{B}\right],
$$

in which the matrices $\left[\underline{M}^{B}\right],\left[\underline{D}^{B}\right]$ and $\left[\underline{K}^{B}\right]$ of the reduced mean system are replaced by the random matrices $\left[\mathbf{M}^{B}\right],\left[\mathbf{D}^{B}\right]$ and $\left[\mathbf{K}^{B}\right]$ defined on a probability space $(\Theta, \mathcal{T}, \mathcal{P})$ and belonging to an ensemble of matrices (See [2]) such that

$$
\begin{gathered}
{\left[\mathbf{M}^{B}\right],\left[\mathbf{D}^{B}\right],\left[\mathbf{K}^{B}\right] \in \mathbb{M}_{n}^{+}(\mathbb{R}),} \\
E\left\{\left[\mathbf{M}^{B}\right]\right\}=\left[\underline{M}^{B}\right], E\left\{\left[\mathbf{D}^{B}\right]\right\}=\left[\underline{D}^{B}\right] E\left\{\left[\mathbf{K}^{B}\right]\right\}=\left[\underline{K}^{B}\right], \\
E\left\{\left\|\left[\mathbf{M}^{B}\right]^{-1}\right\|_{F}^{2}\right\}<+\infty, E\left\{\left\|\left[\mathbf{D}^{B}\right]^{-1}\right\|_{F}^{2}\right\}<+\infty, E\left\{\left\|\left[\mathbf{K}^{B}\right]^{-1}\right\|_{F}^{2}\right\}<+\infty,
\end{gathered}
$$

in which $E\{$.$\} is the mathematical expectation and where \|\cdot\|_{F}$ is the Frobenius norm defined as $\|A\|_{F}=\left\{\operatorname{tr}\left\{[A]^{*}[A]\right\}\right\}^{1 / 2}=\left\{\sum_{j=1}^{n} \sum_{k=1}^{m}\left|[A]_{j k}\right|^{2}\right\}^{1 / 2}$ with $[A]^{*}=[\bar{A}]^{T}$. The probability distribution of each random matrix $\left[\mathbf{M}^{B}\right],\left[\mathbf{D}^{B}\right]$ and $\left[\mathbf{K}^{B}\right]$ depend respectively on the dispersion parameters $\delta_{M}^{B}$, $\delta_{D}^{B}$ and $\delta_{K}^{B}$ such that

$$
\delta_{P}^{B}=\left(\frac{E\left\{\left\|\left[\mathbf{P}^{B}\right]-\left[\underline{P}^{B}\right]\right\|_{F}^{2}\right\}}{\left\|\left[\underline{P}^{B}\right]\right\|_{F}^{2}}\right)^{\frac{1}{2}}, \text { for } \mathrm{P} \text { in }\{M, D, K\}
$$

The dispersion parameters allow the level of uncertainites to be controlled. In addition, it can be found in (see [1], [2]) an algebraic representation of each random matrix which allows independent realisations to be explicitly constructed useful to solve the random equations by the Monte Carlo method. For each random matrix, this random generator only depends on the mean value, on the dimension of the matrix and on the dispersion parameter $\delta$. Such an approach is used in this paper. 


\subsubsection{Identification of the dispersion parameters}

As explained in 3.1.2, the probability distributions of the random matrices (and then their random generators) depend on the vector $\boldsymbol{\delta}=\left(\delta_{M}^{B}, \delta_{D}^{B}, \delta_{K}^{B}\right)$ of the dispersion parameters which is identified using the reference computational model. The observation of the stochastic simplified computational model is defined similarly to the observation defined by Eq. (8) for the reference computational model. We then introduce the condensed dynamical stiffness matrix $\left[\mathbf{Z}^{B}(\omega)\right]=\left[\mathbf{A}_{c c}^{B}(\omega)\right]-\left[\mathbf{A}_{c y}^{B}(\omega)\right]\left[\mathbf{A}_{y y}^{B}(\omega)\right]^{-1}\left[\mathbf{A}_{y c}^{B}(\omega)\right]$ of the linear subsystem on the coupling interface. Taking into account the properties of the probabilistic model, it can be shown that for all $\omega$ fixed in the frequency band $\mathcal{B}$, the random matrix $\left[\mathbf{Z}^{B}(\omega)\right]$ is invertible almost surely and that the random variable $J(\boldsymbol{\delta})$ defined by

$$
J(\delta)=\int_{\mathcal{B}}\left\|\left[\mathbf{Z}^{B}(\omega)\right]^{-1}\right\|_{F}^{2} d \omega
$$

exists and has a finite mean value. It should be noted that the random variable $J$ depends on $\delta$ because the probability distributions of the random matrices $\left[\mathbf{M}^{B}\right],\left[\mathbf{D}^{B}\right]$ and $\left[\mathbf{K}^{B}\right]$ depend on $\boldsymbol{\delta}$. Let $x \mapsto p_{J}(x, \boldsymbol{\delta})$ be the probabilty distribution of the random variable $J(\boldsymbol{\delta})$ with respect to $d x$. For any $x$ fixed in $\left[0,+\infty\right.$ [ and for any value of the vector $\delta$ in the admissible space $\mathcal{C}_{a d}$ of the dispersion parameters, the value $p_{J}(x, \boldsymbol{\delta})$ of the probability density function is estimated by using the above proabilistic model and the Monte Carlo simulation. Note that the corresponding deterministic value of $J(\boldsymbol{\delta})$ for the reference computational model is denoted by $J^{\text {ref }}$ given by Eq. (8). The method used to identify vector $\boldsymbol{\delta}$ is the maximum likelihood method (see for instance [4]) for the random observation $J(\boldsymbol{\delta})$. We then have to solve the following optimisation problem

$$
\boldsymbol{\delta}^{o p t}=\arg \max _{\boldsymbol{\delta} \in \mathcal{C}_{a d}}\left(p_{J}\left(J^{r e f} ; \boldsymbol{\delta}\right)\right),
$$

in which $\boldsymbol{\delta}^{\text {opt }}$ is the identified value of vector $\boldsymbol{\delta}$.

3.2 Random dynamical transient response of the stochastic non-linear simplified computational model

For the stochastic system, the displacement vector of the stochastic linear subsystem $\Omega_{B}$ is denoted by $\left(\mathbf{U}_{p}^{B}(t), \mathbf{U}_{c}^{B}\right)(t)$ in which $\mathbf{U}_{p}^{B}(t)$ is with values in $\mathbb{R}^{n_{p}}$ and $\mathbf{U}_{c}^{B}(t)$ is with values in $\mathbb{R}^{n_{c}}$. The displacement vector of the stochastic non-linear subsystem $\Omega_{A}$ is denoted by $\left(\mathbf{U}_{p}^{A}(t), \mathbf{U}_{c}^{A}(t)\right)$ in which $\mathbf{U}_{p}^{A}(t)$ is with values in $\mathbb{R}^{n_{p}^{\prime}}$ and $\mathbf{U}_{c}^{A}(t)$ is with values in $\mathbb{R}^{n_{c}}$. Taking into account Eq. (2.2), these random vectors are written as

$$
\left[\begin{array}{c}
\mathbf{U}_{p}^{A}(t) \\
\mathbf{U}_{c}^{A}(t)
\end{array}\right]=\left[H^{A}\right]\left[\begin{array}{c}
\mathbf{Y}^{A}(t) \\
\mathbf{U}_{c}^{A}(t)
\end{array}\right], \quad\left[\begin{array}{c}
\mathbf{U}_{p}^{B}(t) \\
\mathbf{U}_{c}^{B}(t)
\end{array}\right]=\left[H^{B}\right]\left[\begin{array}{c}
\mathbf{Y}^{B}(t) \\
\mathbf{U}_{c}^{B}(t)
\end{array}\right],
$$

in which $\mathbf{Y}^{A}(t)$ is the $\mathbb{R}^{N^{\prime}}$-vector random generalized coordinates of the stochastic non-linear subsystem $\Omega_{A}$ with fixed interface and where $\mathbf{Y}^{B}(t)$ is the $\mathbb{R}^{N}$-vector random generalized coordinates of the stochastic linear subsystem $\Omega_{B}$ with fixed interface. The coupling of the stochastic linear subsystem $\Omega_{B}$ with the non-linear subsystem $\Omega_{A}$ is carried out in writting the continuity of the random displacement $\mathbf{U}^{c}(t)=\mathbf{U}_{c}^{A}(t)=\mathbf{U}_{c}^{B}(t)$ on the coupling interface $\Gamma$ and in writting the equilibrium of the coupling interface in terms of forces applied to the two subsystems (external forces $\tilde{\mathbf{f}}_{c}(t)$ applied to the coupling interface, forces $\left[S^{A}\right]^{T} \tilde{\mathbf{f}}_{p}^{A}(t)$ and $\left[S^{B}\right]^{T} \tilde{\mathbf{f}}_{p}^{B}(t)$ applied to the coupling interface and due

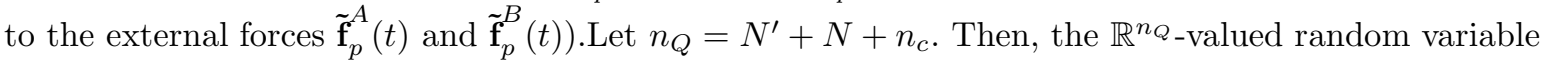
$\mathbf{Q}(t)=\left(\mathbf{Y}^{A}(t), \mathbf{Y}^{B}(t), \mathbf{U}^{c}(t)\right)$ which is composed of the random generalized coordinates $\mathbf{Y}^{A}(t)$ of the random non-linear subsystem, of the random generalized coordinates $\mathbf{Y}^{B}(t)$ of the linear subsystem and of the random coupling DOF $\mathbf{U}_{c}(t)$, is solution of the random non-linear dynamical system

$$
[\tilde{\mathbf{M}}] \ddot{\mathbf{Q}}(t)+[\tilde{\mathbf{D}}] \dot{\mathbf{Q}}(t)+[\tilde{\mathbf{K}}] \mathbf{Q}(t)+\mathcal{F}^{N L}(\mathbf{Q}(t), \dot{\mathbf{Q}}(t))=\mathcal{F}(t) \quad, \quad t \in[0, T]
$$


with the initial conditions

$$
\mathbf{Q}(0)=\dot{\mathbf{Q}}(0)=0,
$$

with

$$
\begin{gathered}
{[\tilde{\mathbf{M}}]=\left[\begin{array}{ccc}
M_{y y}^{A} & 0 & M_{y c}^{A} \\
0 & \mathbf{M}_{y y}^{B} & \mathbf{M}_{y c}^{B} \\
M_{c y}^{A} & \mathbf{M}_{c y}^{B} & M_{c c}^{A}+\mathbf{M}_{c c}^{B}
\end{array}\right],[\tilde{\mathbf{D}}]=\left[\begin{array}{ccc}
D_{y y}^{A} & 0 & D_{y c}^{A} \\
0 & \mathbf{D}_{y y}^{B} & \mathbf{D}_{y c}^{B} \\
D_{c y}^{A} & \mathbf{D}_{c y}^{B} & D_{c c}^{A}+\mathbf{D}_{c c}^{B}
\end{array}\right],} \\
{[\tilde{\mathbf{K}}]=\left[\begin{array}{ccc}
K_{y y}^{A} & 0 & K_{y c}^{A} \\
0 & \mathbf{K}_{y y}^{B} & \mathbf{K}_{y c}^{B} \\
K_{c y}^{A} & \mathbf{K}_{c y}^{B} & K_{c c}^{A}+\mathbf{K}_{c c}^{B}
\end{array}\right],} \\
\mathcal{F}^{N L}(\mathbf{Q}(t), \dot{\mathbf{Q}}(t))=\left[\begin{array}{c}
{\left[\Phi^{A}\right]^{T} \tilde{\mathbf{f}}^{N L}\left(\mathbf{Y}^{A}(t), \dot{\mathbf{Y}}^{A}(t)\right)} \\
0 \\
0
\end{array}\right], \mathcal{F}(t)=\left[\begin{array}{c}
{\left[\Phi^{A}\right]^{T} \tilde{\mathbf{f}}_{p}^{A}(t)} \\
{\left[\Phi^{B}\right]^{T} \tilde{\mathbf{f}}_{p}^{B}(t)} \\
\tilde{\mathbf{f}}_{c}(t)+\left[S^{A}\right]^{T} \tilde{\mathbf{f}}_{p}^{A}(t)+\left[S^{B}\right]^{T} \tilde{\mathbf{f}}_{p}^{B}(t)
\end{array}\right] .
\end{gathered}
$$

\section{Probabilistic model of the stochastic load with model uncertainties}

The transient load $\tilde{\mathbf{f}}(t)=\left(\tilde{\mathbf{f}}_{p}^{A}(t), \tilde{\mathbf{f}}_{p}^{B}(t), \tilde{\mathbf{f}}_{c}(t)\right)$ related to the displacement $\mathbf{u}(t)=\left(\mathbf{u}_{p}^{A}(t), \mathbf{u}_{p}^{B}(t), \mathbf{u}_{c}(t)\right)$ is, for instance, due to the turbulent flow which then induces a stochastic load. Consequently, such a load is modelled by a stochastic process $\{\tilde{\mathbf{F}}(t), t \in \mathbb{R}\}$. Since all the degrees of freedom of the computational model are not excited by this stochastic load, we then introduce the usual projection operator Proj in order to extract the vector $\mathbf{F}(t)=\operatorname{Proj}(\tilde{\mathbf{F}}(t))$ of the non zero random components of the random vector $\tilde{\mathbf{F}}(t)$. Since the probabilistic model developed below will only be a simple representation of the real stochastic load applied to the structure, model uncertainties must be introduced in order to improve the efficiency of the representation which will be used for the identification of this stochastic load. We then take into account these uncertainties in introducing an additional probabilistic model of uncertainties for this stochastic process $\{\mathbf{F}(t), t \in \mathbb{R}\}$ which is then rewritten as $\left\{\mathbf{F}^{\mathrm{unc}}(t), t \in \mathbb{R}\right\}$.

\subsection{Construction of the stochastic load $\mathbf{F}(t)$}

The stochastic load is modelled by a $\mathbb{R}^{m}$-valued second order stationary Gaussian stochastic process $\{\mathbf{F}(t), t \in \mathbb{R}\}$ defined on a probability space $\left(\Theta^{\prime}, \mathcal{T}^{\prime}, \mathcal{P}^{\prime}\right)$ different from the probability space $(\Theta, \mathcal{T}, \mathcal{P})$, indexed by $\mathbb{R}$, centred, mean square continous on $\mathbb{R}$, physically realizable (causal) and whose matrix-valued autocorrelation function $\tau \mapsto\left[\underline{R}_{\mathbf{F}}(\tau)\right]=E\left\{\mathbf{F}(t+\tau) \mathbf{F}(t)^{T}\right\}$ is integrable on $\mathbb{R}$. This stochastic process is then completely defined by its matrix-valued spectral density function $\left[\underline{S}_{\mathbf{F}}(\omega)\right]=(2 \pi)^{-1} \int_{\mathbb{R}} e^{-i \omega \tau}\left[\underline{R}_{\mathbf{F}}(\tau)\right] d \tau$ which is a continuous and integrable function on $\mathbb{R}$ and which is in values in the set of all the positive $(m \times m)$ hermitian matrices. In addition, we will assume that for all $\omega$ in $\mathbb{R}$, the matrix $\left[\underline{S}_{\mathbf{F}}(\omega)\right]$ is with values in the set $\mathbb{M}_{m}^{+}(\mathbb{C})$ of all the positive definite $(m \times m)$ hermitian matrices. Since the stochastic process is assumed to be physically realizable, the matrix valued spectral density function must satisfy the following usual inequality

$$
\int_{\mathbb{R}} \frac{\log \left(\operatorname{det}\left[\underline{S}_{\mathbf{F}}(\omega)\right]\right)}{1+\omega^{2}}>-\infty
$$

In addition, for all $\omega$ in $\mathcal{B} \subset \mathbb{R}$, the matrix valued spectral density function being positive definite, the Hermitian matrix $\left[\underline{S}_{\mathbf{F}}(\omega)\right]$ is invertible and its Cholesky decomposition yields

$$
\left[\underline{S}_{\mathbf{F}}(\omega)\right]=\left[\underline{L}_{\mathbf{F}}(\omega)\right]^{*}\left[\underline{L}_{\mathbf{F}}(\omega)\right],
$$

in which $\left[\underline{L}_{\mathbf{F}}(\omega)\right]$ is an upper triangular matrix in $\mathbb{M}_{m}(\mathbb{C})$. The numerical simulation of independent realizations $\left\{\mathbf{F}\left(t, \theta^{\prime}\right), t \in \mathbb{R}\right\}$ for $\theta^{\prime} \in \Theta^{\prime}$ ( trajectories) can easily be generated by using adapted and wellknown algorithms (see for instance [13]). 
4.2 Construction of the stochastic load $\mathbf{F}^{\mathrm{unc}}(t)$ including a probabilistic model of uncertainties

As explained above, a probabilistic model of uncertainties must be implemented on the stochastic process $\{\mathbf{F}(t), t \in \mathbb{R}\}$ which is rewritten as $\left\{\mathbf{F}^{\text {unc }}(t), t \in \mathbb{R}\right\}$. The objective of this section is to construct the stochastic process $\left\{\mathbf{F}^{\mathrm{unc}}(t), t \in \mathbb{R}\right\}$ derived from the stochastic process $\{\mathbf{F}(t), t \in \mathbb{R}\}$.

\subsubsection{Defining stochastic load $\boldsymbol{F}^{\mathrm{unc}}(t)$}

Since the stochastic process $\{\mathbf{F}(t), t \in \mathbb{R}\}$ is defined by only one parameter (one data) which is its matrix-valued spectral density function $\left[\underline{S}_{\mathbf{F}}\right]=\left\{\left[\underline{S}_{\mathbf{F}}(\omega)\right], \omega \in \mathbb{R}\right\}$, the stochastic process $\{\mathbf{F}(t), t \in \mathbb{R}\}$ is rewritten as $\left.\left\{\mathbf{F}\left(t ; \underline{S}_{\mathbf{F}}\right]\right), t \in \mathbb{R}\right\}$. Therefore, model uncertainties is introduced using the probabilistic parametric approach of data uncertainties consisting in modelling the deterministic function $\left[\underline{S}_{\mathbf{F}}\right]=\left\{\left[\underline{S}_{\mathbf{F}}(\omega)\right], \omega \in \mathbb{R}\right\}$ by a random function with values in $\mathbb{M}_{m}^{+}(\mathbb{C})$ and denoted by $\left[\mathbf{S}_{\mathbf{F}}\right]=$ $\left\{\left[\mathbf{S}_{\mathbf{F}}(\omega)\right], \omega \in \mathbb{R}\right\}$, defined on a probability space $\left(\Theta ", \mathcal{T} ", \mathcal{P}^{\prime \prime}\right)$. Any realization $\left[\mathbf{S}_{\mathbf{F}}\right]\left(\theta^{\prime \prime}\right)$ for $\theta$ " $\in \Theta$ " of the random function $\left[\mathbf{S}_{\mathbf{F}}\right]$ is such that $\left[\mathbf{S}_{\mathbf{F}}\right]\left(\theta^{\prime \prime}\right)=\left\{\left[\mathbf{S}_{\mathbf{F}}(\omega, \theta ")\right], \omega \in \mathbb{R}\right\}$. Consequently, the stochastic process $\left\{\mathbf{F}^{\text {unc }}(t), t \in \mathbb{R}\right\}$ indexed by $\mathbb{R}$ with values in $\mathbb{R}^{m}$, defined on the probability space $\left(\Theta^{\prime}, \mathcal{T}^{\prime}, \mathcal{P}^{\prime}\right) \times\left(\Theta^{\prime \prime}, \mathcal{T}^{\prime \prime}, \mathcal{P}^{\prime \prime}\right)$, is such that, for all $\theta^{\prime} \in \Theta^{\prime}$ and $\theta^{\prime \prime} \in \Theta^{\prime \prime}$,

$$
\mathbf{F}^{\text {unc }}\left(t, \theta^{\prime}, \theta^{\prime \prime}\right)=\mathbf{F}\left(t, \theta^{\prime} ;\left[\mathbf{S}_{\mathbf{F}}\right]\left(\theta^{\prime \prime}\right)\right)
$$

\subsubsection{Construction of the random function $\left[\boldsymbol{S}_{\boldsymbol{F}}\right]$}

The objective of this section is to construct the random function $\left[\mathbf{S}_{\mathbf{F}}\right]$ in using the information theory that is to is using the maximum entropy principle (Shannon 48). In a first step, we then have to define the available information and then we have to apply the maximum entropy principle. The available information concerning the random function $\left[\mathbf{S}_{\mathbf{F}}(\omega)\right], \omega \in \mathbb{R}$ is the following. For all $\omega$ in $\mathbb{R},\left[\mathbf{S}_{\mathbf{F}}(\omega)\right]$ is a random matrix with values in $\mathbb{M}_{m}^{+}(\mathbb{C})$ and by construction, it is written that

$$
E\left\{\left[\mathbf{S}_{\mathbf{F}}(\omega)\right]\right\}=\left[\underline{S}_{\mathbf{F}}(\omega)\right] \quad, \quad \omega \in \mathbb{R} .
$$

Consequently, for all $\omega$ in $\mathbb{R}$, the random matrix $\left[\mathbf{S}_{\mathbf{F}}(\omega)\right]$ is invertible almost surely, which means that for $\mathcal{P} "$-almost $\theta$ " in $\Theta$ ", the matrix $\left[\mathbf{S}_{\mathbf{F}}\left(\omega, \theta^{\prime \prime}\right)\right]^{-1}$ exists. By construction we will impose that the random matrix $\left[\mathbf{S}_{\mathbf{F}}(\omega)\right]^{-1}$ is a second order random variable which means that

$$
E\left\{\left\|\left[\mathbf{S}_{\mathbf{F}}(\omega)\right]^{-1}\right\|^{2}\right\}<+\infty \quad .
$$

Taking into account Eq. (25), for all $\omega$ in $\mathcal{B}$, the random matrix $\left[\mathbf{S}_{\mathbf{F}}(\omega)\right]$ is normalized as follows,

$$
\left[\mathbf{S}_{\mathbf{F}}(\omega)\right]=\left[\underline{L}_{\mathbf{F}}(\omega)\right]^{*}\left[\mathbf{G}_{m}\right]\left[\underline{L}_{\mathbf{F}}(\omega)\right],
$$

in which the random matrix $\left[\mathbf{G}_{m}\right]$ is defined on the probability space $\left(\Theta^{\prime \prime}, \mathcal{T}^{\prime \prime}, \mathcal{P}^{\prime \prime}\right)$ and belongs to the normalized positive-definite ensemble denoted by $S G^{+}$(see [2]). This random matrix which is independent of $\omega$ is such that

$$
\left[\mathbf{G}_{m}\right] \in \mathbb{M}_{m}^{+}(\mathbb{R}) \quad, \quad E\left\{\left[\mathbf{G}_{m}\right]\right\}=\left[I_{m}\right] \quad, \quad E\left\{\left\|\left[\mathbf{G}_{m}\right]^{-1}\right\|_{F}^{2}\right\}<+\infty \quad .
$$

The dispersion of $\left[\mathbf{S}_{\mathbf{F}}\right]$ is independant of $\omega$ and is controlled by dispersion parameter $\delta_{F}$ which is such that

$$
\delta_{F}=\left\{\frac{1}{m} E\left\{\left\|\left[\mathbf{G}_{m}\right]-\left[I_{m}\right]\right\|\right\}_{F}^{2}\right\}^{1 / 2},
$$

and which has to be chosen such that

$$
0<\delta_{F}<\sqrt{(m+1)(m+5)^{-1}} .
$$

From Eqs. (25), (29) and (30), it can be deduced that Eq. (27) holds. In addition, from Eq. (29), we deduce that for all $\omega$ in $\mathcal{B},\left\|\left[\mathbf{S}_{\mathbf{F}}(\omega)\right]^{-1}\right\|<c(\omega)\left\|\left[\mathbf{G}_{m}\right]^{-1}\right\|$ and consequently, $E\left\{\left\|\left[\mathbf{S}_{\mathbf{F}}(\omega)\right]^{-1}\right\|^{2}\right\} \leq$ $c(\omega)^{2} E\left\{\left\|\left[\mathbf{G}_{m}\right]^{-1}\right\|^{2}\right\} \leq c(\omega)^{2} E\left\{\left\|\left[\mathbf{G}_{m}\right]^{-1}\right\|_{F}^{2}\right\}$. Since $E\left\{\left\|\left[\mathbf{G}_{m}\right]^{-1}\right\|_{F}^{2}\right\}<+\infty$, we then deduce that $E\left\{\left\|\left[\mathbf{S}_{\mathbf{F}}(\omega)\right]^{-1}\right\|_{F}^{2}\right\}<+\infty$. 
4.2.3 Properties of stochastic process $\boldsymbol{F}^{\mathrm{unc}}(t)$

For $\mathcal{P}^{\prime \prime}$-almost all $\theta^{\prime \prime}$ in $\Theta^{\prime \prime}$, the mean function of the centred stochastic process $\left.\left\{\mathbf{F}\left(t,\left[\mathbf{S}_{\mathbf{F}}\right]\left(\theta^{\prime \prime}\right)\right), t \in \mathbb{R}\right)\right\}$ is such that

$$
E\left\{\mathbf{F}\left(t,\left[\mathbf{S}_{\mathbf{F}}\right]\left(\theta^{\prime \prime}\right)\right)\right\}=\int_{\Theta^{\prime}} \mathbf{F}\left(t, \theta^{\prime},\left[\mathbf{S}_{\mathbf{F}}\right]\left(\theta^{\prime \prime}\right)\right) d \mathcal{P}^{\prime}\left(\theta^{\prime}\right)=0
$$

and its autocorrelation function is defined by

$$
\left[R_{\mathbf{F}}\left(\tau,\left[\mathbf{S}_{\mathbf{F}}\right]\left(\theta^{\prime \prime}\right)\right)\right]=\int_{\Theta^{\prime}} \mathbf{F}\left(t+\tau, \theta^{\prime},\left[\mathbf{S}_{\mathbf{F}}\right]\left(\theta^{\prime \prime}\right)\right) \mathbf{F}\left(t, \theta^{\prime},\left[\mathbf{S}_{\mathbf{F}}\right]\left(\theta^{\prime \prime}\right)\right)^{T} d \mathcal{P}^{\prime}\left(\theta^{\prime}\right)
$$

From Eqs. (25) and (29) and since the function $\left[\underline{S}_{\mathbf{F}}\right]$ is continuous and integrable on $\mathbb{R}$, we deduce that the matrix valued spectal density function $\left[\mathbf{S}_{\mathbf{F}}\right]\left(\theta^{\prime \prime}\right)=\left\{\omega \mapsto\left[\mathbf{S}_{\mathbf{F}}\left(\omega, \theta^{\prime \prime}\right)\right]\right\}$ which is such that

$$
\left[R_{\mathbf{F}}\left(\tau,\left[\mathbf{S}_{\mathbf{F}}\right]\left(\theta^{\prime \prime}\right)\right)\right]=\int_{\mathbb{R}} e^{i \omega \tau}\left[\mathbf{S}_{\mathbf{F}}\left(\omega, \theta^{\prime \prime}\right)\right] d \omega
$$

is a continuous and integrable function on $\mathbb{R}$. Using the inverse Fourier Transform for integrable functions, we deduce that

$$
\left[\mathbf{S}_{\mathbf{F}}\left(\omega, \theta^{\prime \prime}\right)\right]=\frac{1}{2 \pi} \int_{\mathbb{R}} e^{-i \omega \tau}\left[R_{\mathbf{F}}\left(\tau,\left[\mathbf{S}_{\mathbf{F}}\right]\left(\theta^{\prime \prime}\right)\right)\right] d \tau
$$

(i) Mean function. The mean function of the stochastic process $\left\{\mathbf{F}^{\mathrm{unc}}(t), t \in \mathbb{R}\right\}$ is such that, for all $t$ fixed in $\mathbb{R}$,

$$
E\left\{\mathbf{F}^{\mathrm{unc}}(t)\right\}=\int_{\Theta^{\prime}} \int_{\Theta^{\prime \prime}} \mathbf{F}\left(t, \theta^{\prime},\left[\mathbf{S}_{\mathbf{F}}\right]\left(\theta^{\prime \prime}\right)\right) d \mathcal{P}^{\prime}\left(\theta^{\prime}\right) d \mathcal{P}^{\prime \prime}\left(\theta^{\prime \prime}\right)
$$

Taking into account Eq. (33), it can then be deduced that $E\left\{\mathbf{F}^{\text {unc }}(t)\right\}=0$ and consequently, the stochastic process is centred.

(ii) Autocorrelation function. The autocorrelation function of the stochastic process $\left\{\mathbf{F}^{\mathrm{unc}}(t), t \in \mathbb{R}\right\}$ is such that, for all $t$ and $\tau$ fixed in $\mathbb{R}$,

$$
\begin{aligned}
R_{\mathbf{F}^{\text {unc }}}(t+\tau, t) & =E\left\{\mathbf{F}^{\mathrm{unc}}(t+\tau) \mathbf{F}^{\mathrm{unc}}(t)^{T}\right\} \\
& =\int_{\Theta^{\prime}} \int_{\Theta^{\prime \prime}} \mathbf{F}\left(t+\tau, \theta^{\prime},\left[\mathbf{S}_{\mathbf{F}}\right]\left(\theta^{\prime \prime}\right)\right) \mathbf{F}\left(t, \theta^{\prime},\left[\mathbf{S}_{\mathbf{F}}\right]\left(\theta^{\prime \prime}\right)\right)^{T} d \mathcal{P}^{\prime}\left(\theta^{\prime}\right) d \mathcal{P}^{\prime \prime}\left(\theta^{\prime \prime}\right) .
\end{aligned}
$$

Taking into account Eq. (34), we deduce that $R_{\mathbf{F}^{u n c}}(t+\tau, t)=\int_{\Theta^{\prime \prime}}\left[R_{\mathbf{F}}\left(\tau,\left[\mathbf{S}_{\mathbf{F}}\right]\left(\theta^{\prime \prime}\right)\right)\right] d \mathcal{P}^{\prime \prime}\left(\theta^{\prime \prime}\right)$. Consequently, for all $\tau$ in $\mathbb{R}$, one have

$$
R_{\mathbf{F}^{\text {unc }}}(\tau)=E\left\{\left[R_{\mathbf{F}}\left(\tau,\left[\mathbf{S}_{\mathbf{F}}\right]\right)\right\} .\right.
$$

(iii) Matrix-valued spectral density function. The matrix-valued spectral density function $\left[S_{F^{u n c}}\right]$ of the stochastic process $\left\{\mathbf{F}^{\text {unc }}(t), t \in \mathbb{R}\right\}$ is such that, for all $\tau$ in $\mathbb{R}$,

$$
R_{\mathbf{F}^{\text {unc }}}(\tau)=\int_{\mathbb{R}} e^{i \omega \tau}\left[S_{\mathbf{F}^{\text {unc }}}(\omega)\right] d \omega
$$

Equation (39) can be rewritten as

$$
R_{\mathbf{F}}^{\text {unc }}(\tau)=\int_{\Theta^{\prime \prime}}\left[R_{\mathbf{F}}\left(\tau,\left[\mathbf{S}_{\mathbf{F}}\right]\left(\theta^{\prime \prime}\right)\right)\right] d \mathcal{P}^{\prime \prime}\left(\theta^{\prime \prime}\right)
$$

and using Eq. (35) yields

$$
R_{\mathbf{F}}^{\text {unc }}(\tau)=\int_{\Theta^{\prime \prime}} \int_{\mathbb{R}} e^{i \omega \tau}\left[\mathbf{S}_{\mathbf{F}}\left(\omega, \theta^{\prime \prime}\right)\right] d \omega d \mathcal{P}^{\prime \prime}\left(\theta^{\prime \prime}\right)=\int_{\mathbb{R}} e^{i \omega \tau} E\left\{\left[\mathbf{S}_{\mathbf{F}}(\omega)\right]\right\} d \omega
$$

Equation (27), Eq. (42) yields $\left.R_{\mathbf{F}^{\text {unc }}}(\tau)=\int_{\mathbb{R}} e^{i \omega \tau}\left[\underline{S}_{\mathbf{F}}(\omega)\right]\right\} d \omega$ which proves that

$$
\left[S_{\mathbf{F}^{\text {unc }}}(\omega)\right]=E\left\{\left[\mathbf{S}_{\mathbf{F}}(\omega)\right]\right\}=\left[\underline{S}_{\mathbf{F}}(\omega)\right] .
$$


The last equation proves that for all $\omega \in \mathbb{R}$, the matrix $\left[S_{\mathbf{F}^{\text {unc }}}(\omega)\right]$ belongs to $\mathbb{M}_{m}^{+}(\mathbb{C})$.

(iv) Second-order stochastic process. Since $\left[\underline{S}_{\mathbf{F}}\right]$ is an integrable function on $\mathbb{R}$ and from Eqs. (40) and (43), it can be deduced that

$$
E\left\{\left\|\mathbf{F}^{\mathrm{unc}}(t)\right\|^{2}\right\}=\operatorname{tr} \int_{\mathbb{R}}\left[\underline{S}_{\mathbf{F}}(\omega)\right] d \omega<+\infty .
$$

which proves that $\left.\left\{\mathbf{F}^{\mathrm{unc}}(t), t \in \mathbb{R}\right)\right\}$ is a second-order stochastic process.

(v) Realizability, probability distribution and stationarity. From Eqs. (24) and (43), it can be deduced that the stochastic process $\left.\left\{\mathbf{F}^{\mathrm{unc}}(t), t \in \mathbb{R}\right)\right\}$ is physically realizable. For $\mathcal{P}^{\prime \prime}$-almost all $\theta^{\prime \prime}$ in $\Theta^{\prime \prime}$, the stochastic process $\left.\left\{\mathbf{F}\left(t,\left[\mathbf{S}_{\mathbf{F}}\right]\left(\theta^{\prime \prime}\right)\right), t \in \mathbb{R}\right)\right\}$ is Gaussian but it can easily be verified that the stochastic process $\left.\left\{\mathbf{F}^{\mathrm{unc}}(t), t \in \mathbb{R}\right)\right\}$ is not a Gaussian stochastic process. Since the mean function of the second-order stochastic process $\left.\left\{\mathbf{F}^{\mathrm{unc}}(t), t \in \mathbb{R}\right)\right\}$ is independent of $t$ (centred process) and since its autocorrelation function depends only on $\tau$, it can be concluded that the stochastic process $\left.\left\{\mathbf{F}^{\mathrm{unc}}(t), t \in \mathbb{R}\right)\right\}$ is mean-square stationary. However, since the stochastic process is not Gaussian, it cannot directly be deduced from the mean-square stationarity that the stochastic process is stationary. Nevertheless, it can be proved that the stochastic process $\left.\left\{\mathbf{F}^{\mathrm{unc}}(t), t \in \mathbb{R}\right)\right\}$ is in fact stationary.

\subsubsection{Generator of independent trajectories of the stochatic process $\left.\left\{\boldsymbol{F}^{\mathrm{unc}}(t), t \in \mathbb{R}\right)\right\}$}

The stochastic problem defined in Section 3.2 will be solved by the Monte Carlo simulation method. Consequently, we need to construct a generator of independent trajectories of the stochatic process $\left.\left\{\mathbf{F}^{\mathrm{unc}}(t), t \in \mathbb{R}\right)\right\}$, that is to say to construct for all $\left(\theta^{\prime}, \theta^{\prime \prime}\right) \in \Theta^{\prime} \times \Theta^{\prime \prime}$, the trajectory $\left\{\mathbf{F}^{\mathrm{unc}}\left(t, \theta^{\prime}, \theta^{\prime \prime}\right), t \in\right.$ $\mathbb{R})\}$ which can be rewritten, taking into account Eq. $(26)$, as $\mathbf{F}^{\mathrm{unc}}\left(t, \theta^{\prime}, \theta^{\prime \prime}\right)=\mathbf{F}\left(t, \theta^{\prime} ;\left[\mathbf{S}_{\mathbf{F}}\right]\left(\theta^{\prime \prime}\right)\right)$. This last equation shows that firstly we have to construct independent realizations $\omega \mapsto\left[\mathbf{S}_{\mathbf{F}}\left(\omega, \theta^{\prime \prime}\right)\right]$ of the random function $\left[\mathbf{S}_{\mathbf{F}}\right]$ and secondly, for a given matrix-valued spectral density function $\omega \mapsto[S(\omega)]=$ $\left[\mathbf{S}_{\mathbf{F}}\left(\omega, \theta^{\prime \prime}\right)\right]$, we have to construct independent trajectories $\left\{\mathbf{F}\left(t, \theta^{\prime} ;[S]\right), t \in \mathbb{R}\right\}$ of the second-order centred stationary Gaussian stochastic process $\{\mathbf{F}(t), t \in \mathbb{R}\}$ for which the matrix-valued spectral density function is $\{[S(\omega)], \omega \in \mathbb{R}\}$.

(i) Construction of independent realizations of the random function $\left[\boldsymbol{S}_{\boldsymbol{F}}\right]$ The independent realizations of the random function $\left[\mathbf{S}_{\mathbf{F}}\right]$ are constructed using Eq. (29) and the algebraic representation of the random matrix $\left[\mathbf{G}_{m}\right]$ (see [2]) belonging to the ensemble $S G^{+}$and which is independent of $\omega$. For readability of the paper, we briefly recall this algebraic representation. The random matrix $\left[\mathbf{G}_{m}\right]$ is written as $\left[\mathbf{G}_{m}\right]=\left[\mathbf{L}_{m}\right]^{T}\left[\mathbf{L}_{m}\right]$, in which $\left[\mathbf{L}_{m}\right]$ is an upper triangular random matrix with values in $\mathbb{M}_{m}(\mathbb{R})$ such that:

(1) random variables $\left\{\left[\mathbf{L}_{m}\right]_{j j^{\prime}}, j \leq j^{\prime}\right\}$ are independent;

(2) for $j \leq j^{\prime}$, real-valued random variables $\left[\mathbf{L}_{m}\right]_{j j^{\prime}}$ can be written as $\left[\mathbf{L}_{m}\right]_{j j^{\prime}}=\sigma_{m} U_{j j^{\prime}}$ in which $\sigma_{m}=\delta_{F}(m+1)^{-1 / 2}$ and where $U_{j j^{\prime}}$ is a real-valued Gaussian random variable with zero mean and variance equal to 1 ;

(3)for $j=j^{\prime}$, positive-valued random variables $\left[\mathbf{L}_{m}\right]_{j j}$ can be written as $\left[\mathbf{L}_{m}\right]_{j j}=\sigma_{m} \sqrt{2 V_{j}}$ in which $\sigma_{m}=\delta_{F}(m+1)^{-1 / 2}$ and where $V_{j}$ is a positive-valued gamma random variable whose probabilty density function $p_{V_{j}}(v)$ with respect to $d v$ is written as

$$
p_{V_{j}}(v)=1_{\mathbb{R}^{+}}(v) \frac{1}{\Gamma\left(\frac{m+1}{2 \delta_{F}^{2}}+\frac{1-j}{2}\right)} v^{\frac{m+1}{2 \delta_{F}^{2}}-\frac{1+j}{2}} e^{-v}
$$

in which $\delta_{F}$ is the dispersion parameter defined by Eq. (31).

(ii) Construction of independent realizations of the trajectories of the stochastic process $\{\boldsymbol{F}(t ;[S]), t \in$ $\mathbb{R}\}$. The numerical simulation of independent realizations $\left\{\mathbf{F}\left(t, \theta^{\prime} ;[S]\right), t \in \mathbb{R}\right\}$ for $\theta^{\prime} \in \Theta^{\prime}$ can easily be generated by using the same algorithms that the one referenced in Section 4.1 (see [13] and [14]).

\section{Identification of the stochastic load}

This section is devoted to the identification of the stochastic load $\left.\left\{\mathbf{F}^{\mathrm{unc}}(t), t \in \mathbb{R}\right)\right\}$ defined and studied in Section 4 using the random responses of the stochastic simplified model of the structure excited by 
this stochastic loads and defined is Section 3 . This identification consists in identifying the mean value $\left[\underline{S}_{\mathbf{F}}(\omega)\right]$ of the matrix-valued spectral density function and the parameter $\delta_{F}$ that controls the level of uncertainties. This stochastic inverse problem is formulated as a stochastic optimization problem.

\subsection{Stochastic equation for simulation of responses}

We have to identify the stochastic load $\mathbf{F}^{\text {unc }}$ in presence of uncertainties in the linear subsystem $\Omega_{\text {simpl }}^{B}$ and in presence of the model uncertainties in the stochastic load. Such an identification is performed using the stochastic equation deduced from Eqs. (18) and (19) in which the deterministic load $\tilde{\mathbf{f}}(t)$ is replaced by the stochastic load $\tilde{\mathbf{F}}^{\text {unc }}(t)$ (including uncertainties). In fact, the vector $\tilde{\mathbf{F}}^{\text {unc }}(t)$ which is written as $\mathbf{F}^{\text {unc }}(t)=\operatorname{Proj}\left(\tilde{\mathbf{F}}^{\text {unc }}(t)\right)$ is made up of zero and of non-zero elements which are the components of the vector $\mathbf{F}^{\text {unc }}(t)$. Consequently, this relation can easily be inversed and yields $\tilde{\mathbf{F}}^{\text {unc }}(t)=$ $\operatorname{Lift}\left(\mathbf{F}^{\mathrm{unc}}(t)\right)$. Let $n_{\mathbb{U}}=n_{p}^{\prime}+n_{p}+n_{c}$ be the total number of DOF for the complete simplified stochastic

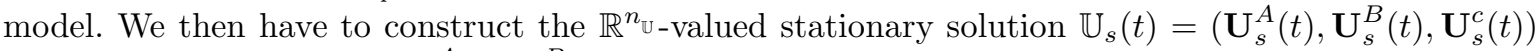
(corresponding to $\left.\mathbb{U}(t)=\left(\mathbf{U}_{p}^{A}(t), \mathbf{U}_{p}^{B}(t), \mathbf{U}^{c}(t)\right)\right)$ verifying the following stochastic equation

$$
\left[\mathbb{U}_{s}(t)\right]=[H]\left[\mathbf{Q}_{s}(t)\right],
$$

in which subindex $s$ is relative to the stationary solution, where the matrix $[H]$ is the assemblage of $\left[H^{A}\right]$ and $\left[H^{B}\right]$, and where the stationary stochastic process $\left\{\mathbf{Q}_{s}(t), t \in \mathbb{R}\right\}$ satisfies, for all $t \in \mathbb{R}$, the stochastic equation

$$
[\tilde{\mathbf{M}}] \ddot{\mathbf{Q}}_{s}(t)+[\tilde{\mathbf{D}}] \dot{\mathbf{Q}}_{s}(t)+[\tilde{\mathbf{K}}] \mathbf{Q}_{s}(t)+\mathbf{f}^{N L}\left(\mathbf{Q}_{s}(t), \dot{\mathbf{Q}}_{s}(t)\right)=[H]^{T} \tilde{\mathbf{F}}^{\mathrm{unc}}(t),
$$

in which $\dot{\mathbf{Q}}_{s}(t)$ and $\ddot{\mathbf{Q}}_{s}(t)$ are the mean-square first and second derivative of $\mathbf{Q}_{s}(t)$. For the identification of the parameters $\left[\underline{S}_{\mathbf{F}}\right]$ and $\delta_{F}$ of the stationary stochastic process $\left\{\tilde{\mathbf{F}}^{\text {unc }}(t), t \in \mathbb{R}\right\}$, we introduce the $\mathbb{R}^{\mu}$-valued random variable $\mathbf{Z}_{s}(t)=\left(Z_{s, 1}(t), \ldots, Z_{s, \mu}(t)\right)$ which represents the observations of the simplified stochastic model made up of components of the vector-valued random response $\mathbb{U}_{s}(t)$. Thus there exists a projection Proj' from $\mathbb{R}^{n_{\mathbb{U}}}$ into $\mathbb{R}^{\mu}$ such that $\mathbf{Z}_{s}(t)=\operatorname{Proj}^{\prime}\left(\mathbb{U}_{s}(t)\right)$. Then, for all $\left(\theta, \theta^{\prime \prime}\right) \in \Theta \times \Theta^{\prime \prime}$, the stationary stochastic process $\left\{\mathbf{Z}_{s}\left(t, \theta, \theta^{\prime \prime}\right), t \in \mathbb{R}\right\}$ is such that $\mathbf{Z}_{s}\left(t, \theta, \theta^{\prime \prime}\right)=\operatorname{Proj}^{\prime}\left([H] \mathbf{Q}_{s}\left(t, \theta, \theta^{\prime \prime}\right)\right)$, where the stationary stochastic process $\left\{\mathbf{Q}_{s}\left(t, \theta, \theta^{\prime \prime}\right), t \in \mathbb{R}\right\}$ is such that, for all $t$ fixed in $\mathbb{R}$, one has

$$
\begin{aligned}
{[\tilde{\mathbf{M}}(\theta)] \ddot{\mathbf{Q}}_{s}\left(t, \theta, \theta^{\prime \prime}\right) } & +[\tilde{\mathbf{D}}(\theta)] \dot{\mathbf{Q}}_{s}\left(t, \theta, \theta^{\prime \prime}\right)+[\tilde{\mathbf{K}}(\theta)] \mathbf{Q}_{s}\left(t, \theta, \theta^{\prime \prime}\right) \\
& +\mathbf{f}^{N L}\left(\mathbf{Q}_{s}\left(t, \theta, \theta^{\prime \prime}\right), \dot{\mathbf{Q}}_{s}\left(t, \theta, \theta^{\prime \prime}\right)\right)=[H]^{T} \operatorname{Lift}\left(\mathbf{F}\left(t ;\left[\mathbf{S}_{\mathbf{F}}\right]\left(\theta^{\prime \prime}\right)\right)\right),
\end{aligned}
$$

where $\left\{\mathbf{F}\left(t ;\left[\mathbf{S}_{\mathbf{F}}\right]\left(\theta^{\prime \prime}\right)\right), t \in \mathbb{R}\right\}$ is a second-order centred stationary Gaussian stochastic process defined in Section 4.2.1.

The next section is devoted to the identification of $\left[\underline{S}_{\mathbf{F}}\right]$ and $\delta_{F}$. In order to perform this identification, we need to introduce two observations relative to the stochastic equation and which are useful to construct the cost functions.

(i) For all $\left(\theta, \theta^{\prime \prime}\right) \in \Theta \times \Theta^{\prime \prime}$, the matrix-valued spectral density function $\left\{\left[\mathbf{S}_{\mathbf{Z}_{s}}\left(\omega, \theta, \theta^{\prime \prime}\right)\right], \omega \in \mathbb{R}\right\}$ of the stationary stochastic process $\left\{\mathbf{Z}_{s}\left(t, \theta, \theta^{\prime \prime}\right), t \in \mathbb{R}\right\}$ is estimated using the periodogram method (ref). One then define the first observation as the random variable $J_{s}$ such that

$$
J_{s}\left(\theta, \theta^{\prime \prime}\right)=\int_{\mathcal{B}}\left\|\left[\mathbf{S}_{\mathbf{Z}_{s}}\left(\omega, \theta, \theta^{\prime \prime}\right)\right]\right\|_{F}^{2} d \omega
$$

Generating $\nu_{\theta}$ independent realizations of the random matrices $[\tilde{\mathbf{M}}],[\tilde{\mathbf{D}}]$ and $[\tilde{\mathbf{K}}]$ and $\nu_{\theta^{\prime \prime}}$ independent realizations of the random function $\left\{\left[\mathbf{S}_{\mathbf{F}}(\omega)\right], \omega \in \mathbb{R}\right\}$, the probabilty distribution $x \mapsto p_{J_{s}}\left(x ; \delta_{F}\right)$ of the random variable $J_{s}$ with respect to $d x$ is estimated by the Monte Carlo simulation method.

(ii) Let $\mathbf{Z}_{s}^{\prime}(t)=\left(Z_{s, 1}^{\prime}(t), \ldots, Z_{s, \mu}^{\prime}(t)\right)$ be the $\mathbb{R}^{\mu}$-valued random variable which is such that for all $\theta \in \Theta$, the stationary stochastic process $\left\{\mathbf{Z}_{s}^{\prime}(t, \theta), t \in \mathbb{R}\right\}$ is such that $\mathbf{Z}_{s}^{\prime}(t, \theta)=\operatorname{Proj}^{\prime}\left([H] \mathbf{Q}_{s}^{\prime}(t, \theta)\right)$, where the stationary stochastic process $\left\{\mathbf{Q}_{s}^{\prime}(t, \theta), t \in \mathbb{R}\right\}$ is such that, for all $t$ fixed in $\mathbb{R}$, one has

$$
\begin{aligned}
{[\tilde{\mathbf{M}}(\theta)] \ddot{\mathbf{Q}}_{s}^{\prime}(t, \theta) } & +[\tilde{\mathbf{D}}(\theta)] \dot{\mathbf{Q}}_{s}^{\prime}(t, \theta)+[\tilde{\mathbf{K}}(\theta)] \mathbf{Q}_{s}^{\prime}(t, \theta) \\
& +\mathbf{f}^{N L}\left(\mathbf{Q}_{s}^{\prime}(t, \theta), \dot{\mathbf{Q}}_{s}^{\prime}(t, \theta)\right)=[H]^{T} \operatorname{Lift}\left(\mathbf{F}\left(t ;\left[\underline{S}_{\mathbf{F}}\right]\right)\right)
\end{aligned}
$$


in which $\left.\left\{\mathbf{F}\left(t ; \underline{S}_{\mathbf{F}}\right]\right), t \in \mathbb{R}\right\}$ is the process defined in Section 4.1. Therefore, for all $\theta \in \Theta$, the matrixvalued spectral density function $\left\{\left[\mathbf{S}_{\mathbf{Z}_{s}^{\prime}}(\omega, \theta)\right], \omega \in \mathbb{R}\right\}$ can be estimated. Generating $\nu_{\theta}$ independent realizations of the random matrices $[\tilde{\mathbf{M}}],[\tilde{\mathbf{D}}]$ and $[\tilde{\mathbf{K}}]$, the second observation is the matrix-valued spectral density function $\left[S_{\mathbf{Z}_{s}^{\prime}}\right]$ which is estimated by the Monte Carlo simulation method. For all $\omega \in \mathbb{R}$, one has

$$
\left[S_{\mathbf{Z}_{s}^{\prime}}(\omega)\right]=\frac{1}{\nu_{\theta}} \sum_{i=1}^{\nu_{\theta}}\left[\mathbf{S}_{\mathbf{Z}_{s}^{\prime}}\left(\omega, \theta_{i}\right)\right]
$$

5.2 Identification of the stochastic loads

In practice, the parameter $\left[\underline{S}_{\mathbf{F}}\right]$ which has to be identified is in fact a function $\omega \mapsto\left[\underline{S}_{\mathbf{F}}(\omega)\right]$. This identification will be performed in introducing a parametric representation of this function which is written as

$$
\left[\underline{S}_{\mathbf{F}}(\omega)\right]=[S(\omega, \mathbf{r})] \quad, \quad \omega \in \mathbb{R} \quad, \quad \mathbf{r} \in \mathcal{C}_{r} \quad,
$$

in which $\mathcal{C}_{r} \subset \mathbb{R}^{\nu_{r}}$ is the admissible set of the parameter $\mathbf{r}$ with values in $\mathbb{R}^{\nu_{r}}$ where $\nu_{r}$ is the number of unknown scalar parameters which have to be identified and where $(\omega, \mathbf{r}) \mapsto[S(\omega, \mathbf{r})]$ is a given function from $\mathbb{R} \times \mathbb{R}^{\nu_{r}}$ into $\mathbb{M}_{m}^{+}(\mathbb{C})$. Therefore, the identification of the stochastic load $\left.\left\{\mathbf{F}^{\text {unc }}(t), t \in \mathbb{R}\right)\right\}$ consists in identifying the $\mathbb{R}^{\nu_{r}+1}$-valued vector $\mathbf{r} '=\left(\mathbf{r}, \delta_{F}\right)$. Let $\left\{\mathbf{Z}_{s}^{\exp }(t)=\left(Z_{s, 1}^{\exp }(t), \ldots, Z_{s, \mu}^{\exp }(t)\right), t \in \mathbb{R}\right\}$ be the $\mathbb{R}^{\mu}$-valued stationary stochastic process which is measured for the manufactured real system and corresponding to the observation stochastic process $\left\{\mathbf{Z}_{s}(t), t \in \mathbb{R}\right\}$. The matrix-valued spectral density

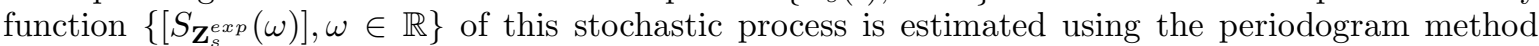
(ref). Therefore, we introduce the variable $J_{s}^{\exp }$ which is such that

$$
J_{s}^{\exp }=\int_{\mathcal{B}} \|\left[S_{\left.\mathbf{Z}_{s}^{\exp }(\omega)\right] \|_{F}^{2} d \omega}\right.
$$

Then, the stochastic loads is estimated in 2 steps:

(i) Estimation of the parameter $\boldsymbol{r}$.

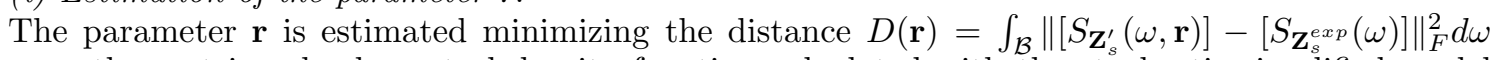
between the matrix-valued spectral density function calculated with the stochastic simplified model and the experimental matrix-valued spectral density function. We then have to solve the following optimization problem

$$
\mathbf{r}_{o p t}=\underset{\mathbf{r} \in \mathcal{C}_{r}}{\arg \min } D(\mathbf{r})
$$

in which $\mathbf{r}_{\text {opt }}$ is the identified value of the vector $\mathbf{r}$.

(ii) Estimation of the dispersion parameter $\delta_{F}$.

The dispersion parameter $\delta_{F}$ is estimated using the maximum likelihood method relative to the random variable $J_{s}$ for which only one experimental value $J_{s}^{\exp }$ is available, i.e,

$$
\delta_{F}^{o p t}=\arg \max _{\delta_{F} \in \mathcal{C}_{\delta_{F}}}\left(p_{J_{s}}\left(J_{s}^{\exp } ; \delta_{F}\right)\right)
$$

in which $\delta_{F}^{\text {opt }}$ is the identified value of the variable $\delta_{F}$ and $\mathcal{C}_{\delta_{F}}$ is the admissible set for the dispersion parameter $\delta_{F}$.

\section{Application}

In this section a numerical simulation of a simple example which is representative of industrial systems is presented in order to validate the methodology developed in this paper. 
6.1 Data for the reference computational model

The reference computational model is made up of one linear subsystem and one non-linear subsystem. The linear subsystem is made up of four parallel Euler beams fixed at their ends. The non-linear subsytem is made up of a beam fixed at its ends, parallel to the other beams and with one transverse symmetric elastic stop (two identical tranverse stops). The five beams are linked by three transversal grids, each grid being modelled by four transverval springs (see Fig. 2). Therefore, the coupling interface between the two subsytems is composed of three points located in the neutral fiber of the beam of the non-linear subsystem. Each beam has a constant circular section with radius $0.5 \mathrm{~m}$, thickness $0.2 \mathrm{~m}$, length $16 \mathrm{~m}$, mass density $250 \mathrm{~kg} / \mathrm{m}^{3}$, Young's modulus $450 \mathrm{~N} / \mathrm{mm}^{2}$ and dampding rate 0.02 . The Young's modulus of the beam of the non-linear subsystem is $750 \mathrm{~N} / \mathrm{mm}^{2}$. The elastic stops are localized at $6 \mathrm{~m}$ from the left fixed end, the gap of each stop is $1.5 \cdot 10^{-6} \mathrm{~m}$ and the choc stiffness is $10^{8} \mathrm{~N} / \mathrm{m}$. The stiffness of each spring of the tranversal grid is $4.10^{7} \mathrm{~N} / \mathrm{m}$. Each beam is modelled by eight beam Euler finite elements of equal lengths and nine nodes. The DOF of the 2 nodes at the ends of the beam are locked. The twelve springs in the three tranversal grids are modelled by 12 spring elements. We are only interested in the transversal displacements in direction $y$ for the plane $x y$ of the beam of the non-linear subsystem(see Fig. 2). Consequently, each beam has 14 DOF of $y$ translation and $z$ rotation. The beam of the nonlinear subsystem is exited by 7 transversal forces applied following
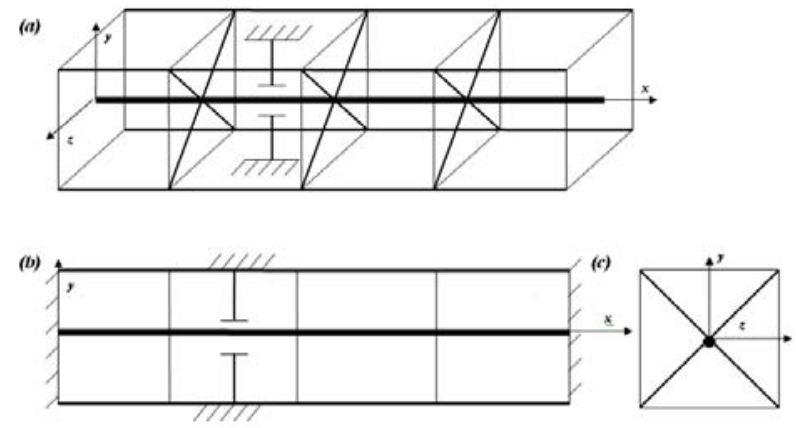

Fig. 2 (a) Reference model:3D view.(b) Transversal view .(c) Tranversale view in the plane of one grid:the 6 diagonal lines represent the 12 springs.

the $y$ direction. The vector of these 7 forces are denoted by $\mathbf{f}^{\mathrm{ref}}$. Then $\left\{\mathbf{f}^{\mathrm{ref}}(t), t \in \mathbb{R}\right\}$ is modeled by a second-order centred stationary Gaussian stochastic process for which its matrix-valued spectral density function $\left[S_{\mathbf{f}^{\text {ref }}}(\omega)\right]$ is such that (1) for all $i$ in $\{1, \ldots, 7\},\left[S_{\mathbf{f}^{\text {ref }}}(\omega)\right]_{i i}$ is a constant equal to $1.3 N^{2} / H z$ on the frequency band of analysis $\mathcal{B}=[-100,100] H z$ and $(2)$ for all $i$ and $j$ in $\{1, \ldots, 7\}$, $\left|\left[S_{\mathbf{f}^{\mathrm{ref}}}(\omega)\right]_{i j}\right|^{2}=\gamma_{i j}(\omega)\left[S_{\mathbf{f}^{\mathrm{ref}}}(\omega)\right]_{i i}\left[S_{\mathbf{f}^{\mathrm{ref}}}(\omega)\right]_{j j}$ where $\gamma_{i j}(\omega)=\exp \left(-\left|x_{i}-x_{j}\right| / \lambda\right)$ in which $\left|x_{i}-x_{j}\right|$ is the distance between the two excited points and $\lambda=4 \mathrm{~m}$ is a reference lenght related to the correlation lengh. In the frequency band of analysis $\mathcal{B}$, there are 21 eigenfrequencies for the linear system made up of the two coupled subsystems without the stops. The first three eigenfrequencies are $5.78 \mathrm{~Hz}, 15.9$ $\mathrm{Hz}$ and $31.1 \mathrm{~Hz}$ and correspond to the eigenmodes for which all the transversal displacements of the five beams are in phase.

\subsection{Data for the simplified computational model}

This part is devoted to the construction of a simplified computational model that will be used to identify the stochastic loads. The simplified computational model consists in replacing the linear substem composed of 4 beams by a linear subsystem composed of an equivalent Euler beam (see Fig. 3). The non-linear subsystem is the same for the 2 models. The section of the equivalent beam is arbitrarily defined and is chosen as a constant circular section with radius $0.5 \mathrm{~m}$, thickness $0.2 \mathrm{~m}$, length $16 \mathrm{~m}$. Its Young's modulus and its the mass density are identified so that the first eigenfrequency of the simplified computational model is the same as the first eigenfrequency of the reference computational 


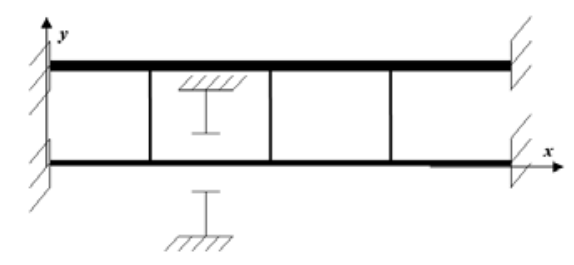

Fig. 3 Simplified model.

model. It should be noted that this choice of simplified model as an equivalent beam does not allow several eigenfrequencies to be correctly fitted. Only the three first eigenfrequencies can correctly be fitted and such a choice induces model uncertainties which are taken into account as explained in Section 3.1.2. Note that this choice of an Euler beam as the equivalent model is representative of the approach chosen in this field of mechanical engineering. It is well understood that a more sophisticated equivalent model could be constructed. The best model would then be a dynamical condensation of the linear subsystem on the coupling interface with the non-linear subsystem. Clearly the objective of this paper is not to proceed with such a dynamical condensation but to test the validity of the use of a rough approximation such as an equivalent Euler beam. After identification, the equivalent beam has a mass density $4 * 250=1000 \mathrm{~kg} / \mathrm{m}^{3}$ and a Young's modulus $4 * 450=1800 \mathrm{~N} / \mathrm{mm}^{2}$. In the frequency band of analysis $\mathcal{B}$, there are 10 eigenfrequencies for the linear system made up of the two coupled subsystems without the stops. The first three eigenfrequencies of the mean simplified computational model are $5.74 \mathrm{~Hz}, 15.3 \mathrm{~Hz}$ and $30.8 \mathrm{~Hz}$ which are to compare to $5.78 \mathrm{~Hz}, 15.9 \mathrm{~Hz}$ and $31.1 \mathrm{~Hz}$ of the reference computational model.

6.3 Comparison between the dynamical response of the reference computational model and the dynamical response of the mean simplified computational model.

For the two models, the stationary stochastic response is calculated in the time interval $[0,220] s$ using an explicit Euler integration scheme for which the time step is... . Let $P_{o b s}$ be the impact point of the non-linear subsystem. The power spectral density function of the stochastic transversal displacement and the stochastic rotation responses in point $P_{\text {obs }}$ (see Fig. 4) is estimated using the periodogram method. It can be seen that the prevision given by the mean simplified computational model is good in the frequency band $[0,30] \mathrm{Hz}$. Nevertheless, there are significant differences in the frequency band $[30,100] H z$ induced by model uncertainties. This is the reason why the model uncertainties are taken into account in order to extend the domain of validity of the mean simplified computational model in the frequency band $[30,100] H z$.

\subsection{System uncertainties modelling and dispersion parameter identification.}

The non-parametric probabilistic model of model uncertainites introduced in Section 3.1.2 is used for stiffness part of the linear subsystem of the mean simplified computational model. We then have to identify the dispersion parameter $\boldsymbol{\delta}=\left(\delta_{K}^{B}\right)$. The estimation of each probability density function in Eq. (17) is carried out with 200 realizations for the Monte Carlo simulation in order to solve stochastic simplified computational model. Fig. 5 shows the likelihood function calculated using Eq. (17) with $\mathcal{C}_{a d}=[0, \sqrt{22 / 34}]$. The maximum is reached for $\boldsymbol{\delta}^{o p t}=0.45$. The confidence region associated with a probability level $P_{c}=0.95$ of the reponse of the stochastic simplified computational model can then be estimated. The calculations are carried out with 100 simulations. The comparison between the reference solution with the response constructed with the stochastic simplified computational model is given in Fig. 6. This figure displays the confidence region of the power spectral density function of the stochastic transversal displacement and the stochastic rotation in point $P_{o b s}$. 


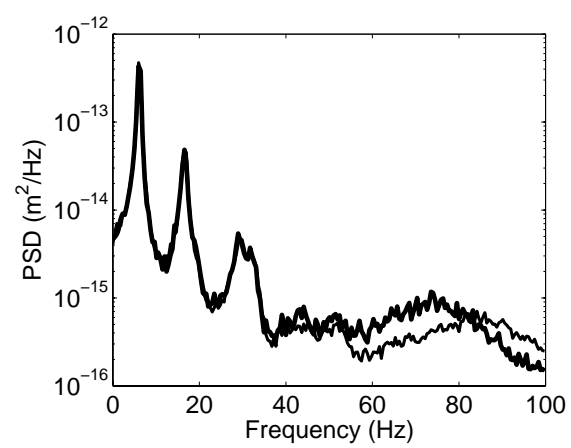

(a)

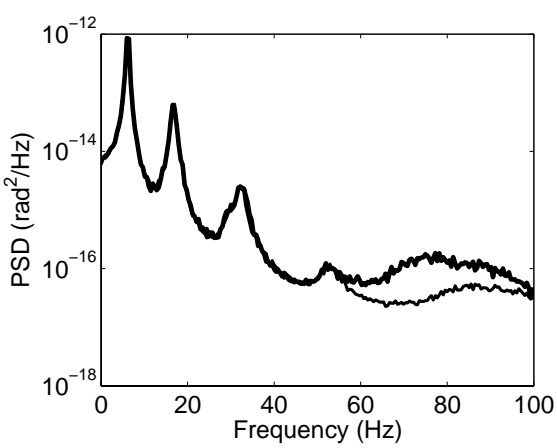

(b)

Fig. 4 Power spectral density function (PSD) for (a) the transversal displacement and (b) the rotation responses. Comparison between the reference computational model in point Pobs (thin line) and the response given by the mean simplified computational model (thick line)..

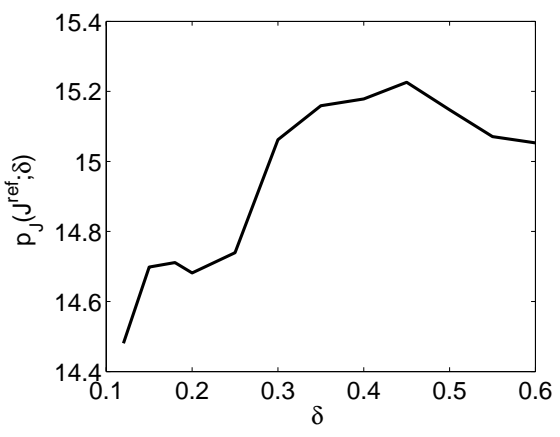

Fig. 5 Graph of function $\delta \mapsto p_{J}\left(J^{r e f} ; \boldsymbol{\delta}\right)$.

6.5 Probabilistic model of the stochastic load $\mathbf{F}(t)$.

We recall that the real model of the stochastic load used to construct the experimental responses in Section 6.4 is now assumed unknown and has to be identified (objective of this paper) using the stochastic simplified computational model. Consequently, we then have to define a model as simple as possible for the stochastic load $\mathbf{F}(t)$ introduced in Section 4.1. We have then chosen to model $\mathbf{F}(t)$ as $\{\mathbf{F}(t)=(T(t), M(t)), t \in \mathbb{R}\}$ in which $T(t)$ is a tranversal force and $M(t)$ a moment applied to the middle of the nonlinear beam (see Fig. 7). This force and this moment are second order centred stationary Gaussian independent stochastic processes. So, they are both completely defined by their power spectral density functions $\left[\underline{S}_{\mathbf{T}}(\omega)\right]$ and $\left[\underline{S}_{\mathbf{M}}(\omega)\right]$. The matrix-valued spectral density function of the stochastic process $\{\mathbf{F}(t), t \in \mathbb{R}\}$ is then defined by

$$
\left[\underline{S}_{\mathbf{F}}(\omega)\right]=\left[\begin{array}{cc}
\underline{S}_{\mathbf{T}}(\omega) & 0 \\
0 & \underline{S}_{\mathbf{M}}(\omega)
\end{array}\right], \quad \omega \in \mathbb{R} .
$$

It is assumed that the function $\omega \mapsto\left[\underline{S}_{\mathbf{F}}(\omega)\right]$ is constant in the frequency band of analysis $\mathcal{B}$ and is such that Eq. (24) is verified. As explained in Section 4.2 , the stochastic process $\left\{\mathbf{F}^{\text {unc }}(t), t \in \mathbb{R}\right\}$ including the probabilistic model of uncertainties is contructed from the stochastic process $\{\mathbf{F}(t), t \in$ $\mathbb{R}\}$. The "experimental" stochastic process $\left\{\mathbf{Z}_{s}^{\exp }(t), t \in \mathbb{R}\right\}$ defined in Section 5.2 is composed of $\mu=7$ stochastic transversal displacements. 


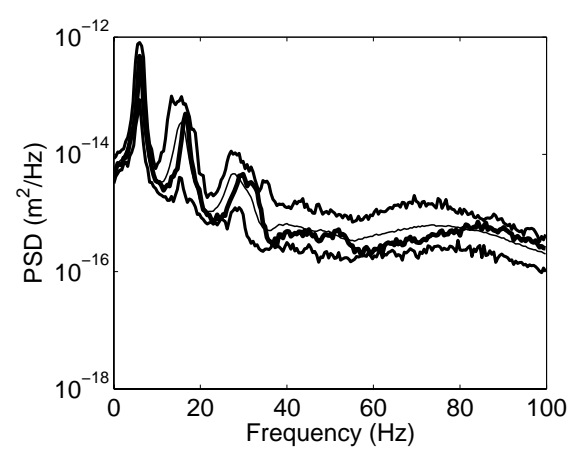

(a)

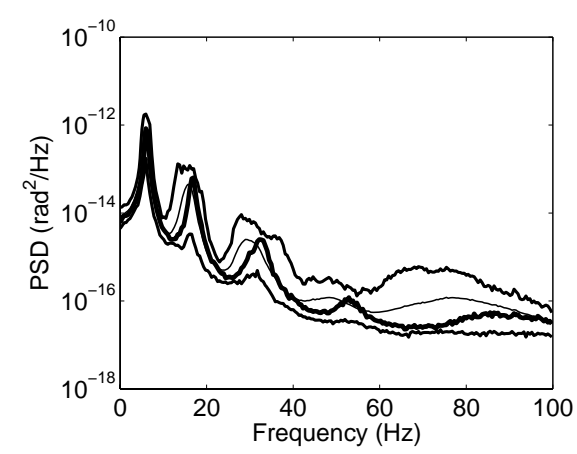

(b)

Fig. 6 Power spectral density function (PSD) for (a) the stochastic transversal displacement and (b) the stochastic rotation in point Pobs: upper and lower envelopes (mid line) and mean response (thin line); reference model (thick line).

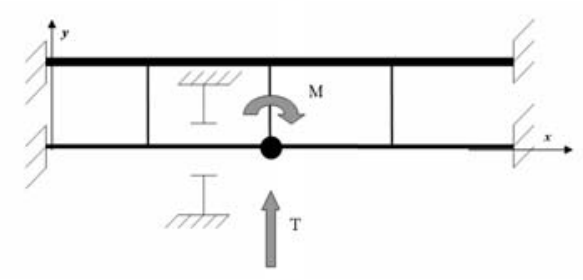

Fig. 7 Definition of the stochastic load.

6.6 Identification of the stochastic load $\mathbf{F}^{\mathrm{unc}}(t)$.

Taking into account Eq. (52), the function $\omega \mapsto\left[\underline{S}_{\mathbf{F}}(\omega)\right]$ which is a constant diagonal hermitian matrix over the frequency band of analysis $\mathcal{B}$ can then be rewritten as

$$
\left[\underline{S}_{\mathbf{F}}(\omega)\right]=[S(\omega, \mathbf{r})]=\left[\begin{array}{cc}
r_{1} & 0 \\
0 & r_{2}
\end{array}\right] \quad, \quad \omega \in \mathcal{B} \quad, \quad \mathbf{r} \in \mathcal{C}_{r}
$$

in which the admissible space $\mathcal{C}_{r}=\left\{\mathbf{r}=\left(r_{1}, r_{2}\right) ; r_{1}>0, r_{2}>0\right\}$. This vector $\mathbf{r}$ is identified using the trial method, consisting in calculating the cost function $D(\mathbf{r})$ for 100 values of the vector $\mathbf{r}$. Then, the optimal value $\mathbf{r}_{\text {opt }}$ defined by Eq. (54) is such that $\mathbf{r}_{o p t}=\left(18 \mathrm{~N}^{2} / \mathrm{Hz}, 20(\mathrm{~N} / \mathrm{m})^{2} / \mathrm{Hz}\right)$. Figure 8 shows the graph of the function $\mathbf{r} \mapsto D(\mathbf{r})$ introduced in Eq. (54). The dispersion paramater $\delta_{F}$ is identified using Eq. (55) with $\left.\mathcal{C}_{\delta_{F}}=\right] 0, \sqrt{3 / 7}\left[\right.$. The maximum of the likelihood function $\delta_{F} \mapsto p_{J_{s}}\left(J_{s}^{\exp } ; \delta_{F}\right)$ is reached for $\delta_{F}^{o p t}=0.04$. Figure 9 compares the reference response with the response constructed with the identified stochastic simplified computational model on which the identified stochastic load is applied.

\section{Conclusions}

The problem under consideration is the identification of a stochastic load applied to a structure through the knowledge of dynamical responses of the structure which has a non-linear behaviour. This identification is performed using a computational non-linear dynamical model of the structure. Since there are both data uncertainties and model uncertainties in the computational model used to perform the identification, the first step of the development consists in introducing a probabilistic model of uncertainties in the structure. In addition, the identification of the stochastic load is carried out using 


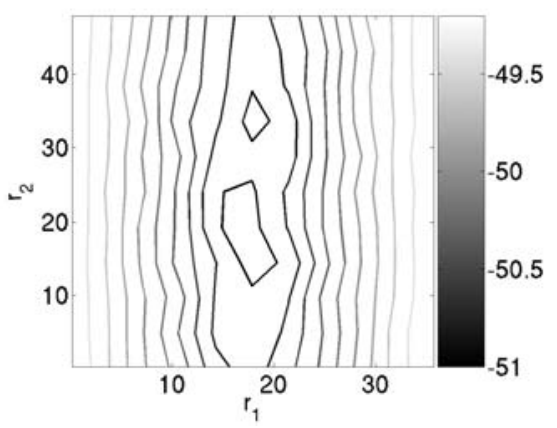

Fig. 8 Graph of the cost function $\left(r_{1}, r_{2}\right) \mapsto D\left(r_{1}, r_{2}\right)$.

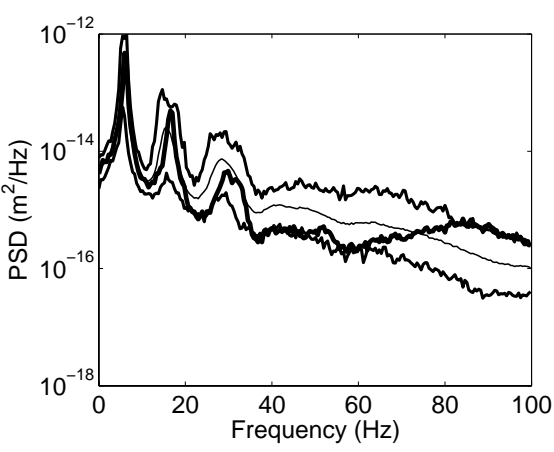

(a)

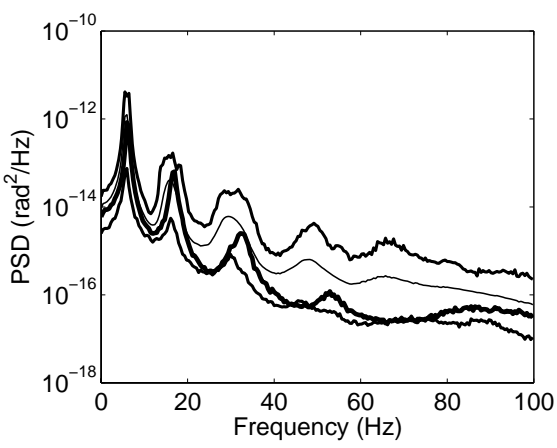

(b)

Fig. 9 Power spectral density function (PSD) for (a) the stochastic transversal displacement and (b) the stochastic rotation in point Pobs: confidence region calculated with the simplified model and the stochastic load: upper and lower envelopes (mid line) and mean response (thin line); reference model (thick line).

a parametric representation of the stochastic process in order that the optimization problem relative to this inverse problem be feasible. The introduction of such a parametric representation induces again model uncertainties on the stochastic loads. The second step of the development then consists in introducing a probabilistic model of uncertainties concerning the stochastic loads. We have then presented a complete methodology to identify the stochastic load taking into account uncertainites in the computational model and in the stochastic loads representation. With respect to the state of the art, this work proposes a new way to perform the experimental identification of a stochastic load with a robust method. The robustness is introduced in taking into account (1) uncertainties in the simplified computational non-linear dynamical model used to carry out this identification and (2) uncertainties in the mathematical representation of the stochastic process which models the loads to be identified. The application presented is representative of a real industrial system and validates the methodology proposed. 


\section{References}

1. C. Soize, A nonparametric model of random uncertainties for reduced matrix models in structural dynamics, Probabilistic Engineering Mechanics,15(3) (2000)277-294.

2. C. Soize, Random matrix theory for modeling uncertainties in computational mechanics, Computer Methods in Applied Mechanics and Engineering,194 (2005) 1333-1366.

3. C. Soize and H. Chebli, Random uncertainties model in dynamic substructuring using a nonparametric probabilistic model, ASCE Journal of Engineering Mechanics,129 (4) 449-457.

4. R.J. Serfling, Approximation Theorems of Mathematical Statistics, John Wiley \& Sons , New York 1980

5. R.R. Craig, Jr. and M.C.C Bampton, Coupling for substructures for dynamic analysis, AIAA Journal, 3 (6), July 1968.

6. G.M. Corcos, Resolution of Pressure in Turbulence, Journal of the Acoustical Society of America, 35, 192-199 1963.

7. S. Granger and L. Perotin, An inverse method for the identification of a distributed random excitation acting on a vibrating structure, part 1 : theory, Mechanical System and Signal Processing, 13 (1), 53-65, 1999.

8. Y. Liu and W.S. Shepard Jr., Dynamic force identification based on enhances least squares and total least-squares schemes in the frequency domain, Journal of sound and vibration, 282 (2005) 37-60.

9. F. Axisa, J. Antunes and B. Villard, Random Excitation of Heat Exchanger Tubes by Cross-Flow, Journal of Fluids and Structures, 4 (1990) 321-342.

10. A.N. Tikhonov and V.Y. Arsenin, Solutions of Ill-Posed Problems, 1997, Wiley, New-York.

11. A. Tarantola, Inverse Problem Theory, 1987, Elsevier, Amsterdam.

12. C.Desceliers, C.Soize, S.Cambier, Nonparametric parametric model for random uncertainties in nonlinear structural dynamics. Application to earthquake engineering, Eartquake Engineering and Structural Dynamics, 33(3), 315-327 (2004).

13. F.Poirion, C.Soize, Numerical methods and mathematical aspects for simulation of homogeneous and non homogeneous Gaussian vector fields, pp. 17-53, in Probabilistic Methods in Applied Physics, SpringerVerlag, Berlin, 1995.

14. M. Shinozuka, C.M. Jan, Digital simulation of random processes and its applications , Journal of sound and vibration, 25 (1) 111-128 (1972).

15. C.Soize, The Fokker-Planck Equation for Stochastic Dynamical Systems and Explicit Steady State Solutions, World Scientific, Singapour, 1994

16. P.Kree, C.Soize, Mathematics of Random Phenomena, Reidel, Dordrecht, 1986. 